\title{
An Uncommon Presentation of Myasthenia Gravis with Thymoma - A Case Report and Review of Literature
}

\author{
MA HANNAN, ${ }^{1}$ MOHAMMAD SAYEED HASSAN, ${ }^{2}$ AYESHA BEGUM,${ }^{3}$ A.K.M. NURUL KABIR ${ }^{4}$
}

\begin{abstract}
:
Myasthenia Gravis (MG) is a rare disease of neuromuscular junction which typically presents with fatigable weakness of cranial and limb muscles. But patient may present with vague symptoms which may mislead physicians to an incorrect diagnosis. We are reporting a 45 year old cobbler presented with the complaints of lack of energy, aching pain in shoulder, back, upper arm and difficulty in swallowing of both solid and liquid food without any diurnal variation for two and half years. He was ultimately diagnosed as a case of MG and thymectomy revealed thymoma.
\end{abstract}

Keyword: Myasthenia Gravis, Thymoma,

\section{Introduction:}

Myasthenia gravis (MG) is an autoimmune disease in which muscular weakness and fatigability of skeletal muscles are caused by an antibody against the acetylcholine receptor (AChR) at the neuromuscular junction. MG is rare, with a prevalence of 50 to 125 cases per million populations. The condition may affect individuals in any age group, with a peak incidence in females in their twenties and thirties; and in males aged between 60 and 70 years. ${ }^{1}$ Generally, immunological myasthenia gravis is sporadic. Familial cases are uncommon and constitute less than $5 \%$ of all the myasthenia gravis patients. ${ }^{2} \mathrm{MG}$ is more common in female in young age. In later life it is male predominant. Progression is rapid in male. Thymoma is common in old males (50-60yrs). ${ }^{3}$ We report a case of Myasthenia Gravis presenting with atypical symptoms.

\section{Case report:}

A 45 year old right handed nondiabetic, normotensive cobbler presented with the complaints of lack of energy, generalized weakness, aching pain in shoulder, back, upper arm and difficulty in swallowing of both solid and liquid food for two and half years without any diurnal variation (figure 1).

For these complaints he consulted a long list of physicians and surgeons including general physician, internist, orthopedic surgeon, ENT specialist who prescribed him several types of medications including vitamins, minerals, NSAIDs, Muscle relaxants, antibiotics, antidepressants. But his condition did not improve and lastly he started to take indigenous medications.

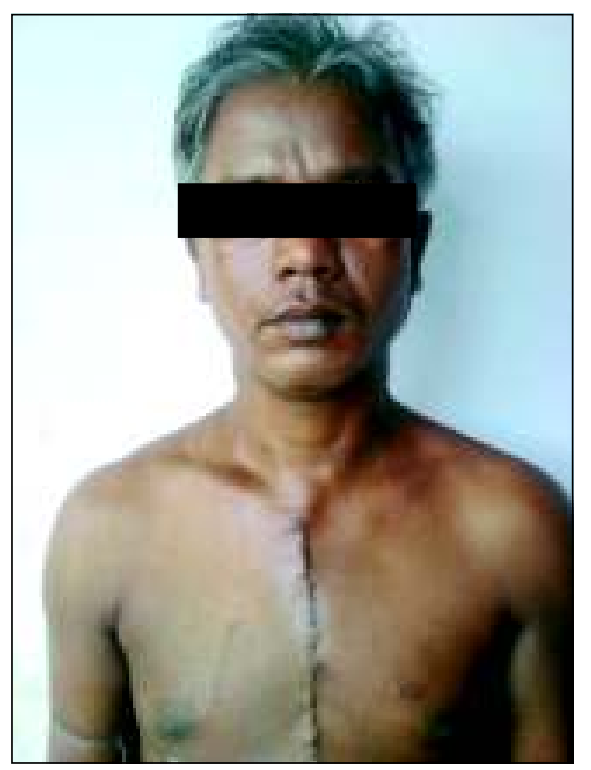

Fig.-1: $M G$ patient with standard median trans-sternal thoracotomy scar.

After consulting with us, he was examined and investigated accordingly. On examination we found partial ptosis though the patient never complained. Ice pack test, ceiling test, counting test and forearm abduction tests were inconclusive.

Routine investigations showed no abnormality. Hb- $11.8 \mathrm{gm} /$ dl,TC of WBC-9000/cmm (N-62\%, L-28\%, E-8\%, B-2\%), Platelet-200000/cmm, ESR- $27 \mathrm{~mm}$ in $1^{\text {st }}$ hour, PBFUnremarkable, RBS- $4.9 \mathrm{mmol} / \mathrm{L}$, S. electrolyte- $\mathrm{Na}^{+}-134 \mathrm{mmol} /$ $\mathrm{L}, \mathrm{K}^{+}-3.6 \mathrm{mmol} / \mathrm{L}$, Urine R/E- normal, Thyroid function test $-\operatorname{Normal}\left(\mathrm{T}_{3}-1.28 \mathrm{ng} / \mathrm{mg}, \mathrm{T}_{4}-8.45 \mathrm{micro} \mathrm{gm} / \mathrm{dl}, \mathrm{TSH}-3.15\right.$

1. Professor of Neurology, BSMMU, Dhaka

2. MD Neurology (final part) student, BSMMU, Dhaka

3. Lecturer of Microbiology, Dhaka Medical College

4. Assistant Professor of Pathology, BSMMU, Dhaka

Correspondence: Dr. M A Hannan, Professor, Department of Neurology, Room no-1304, Block-D, BSMMU, Dhaka. Phone - 01199803587 
$\mathrm{mU} / \mathrm{ml}$ ), CPK- $151 \mathrm{U} / \mathrm{L}, \mathrm{ANA}$ - negative, pANCA- negative, cANCA- negative, Anti-Jo ab- negative, ECG- normal.

Antibodies associated with MG (Anti Ach R Ab, Anti MuSK $\mathrm{Ab}$, Anti skeletal Ab) could not be done. Repetitive Nerve Stimulation test was consistent with Myasthenia Gravis (9.2 to $20.9 \%$ decremental responses). $\mathrm{X}$ ray chest $\mathrm{P}-\mathrm{A}$ and right lateral View; and CT scan of chest were done but there were no evidence of thymic enlargement.

The patient was diagnosed as a case of generalized $\mathrm{MG}$ stage II- A. We started pyridostigmine in low dose which showed significant improvement. The patient was referred to cardiothoracic surgeon for thymectomy. Thymectomy was done on $2^{\text {nd }}$ August, 2010 by standard median trans-sternal thoracotomy.

Histopathological examination showed well capsulated tumour composed of lymphocytes and thymic epithelial cells. No cellular atypia or capsular invasion was seen. It was labeled as WHO classification Type AB (figure 2).



Fig.-2: Thymoma type $A B$

Now the patient is on pyridostigmine $30 \mathrm{mg} 6$ hourly. Clinically he is much improved. He can perform his daily activities without significant physical disability. At present, the patient is under our regular follow up.

\section{Discussion:}

Myasthenia gravis is a potentially serious but treatable organ specific autoimmune disorder characterised by weakness and fatigability of the voluntary muscles caused by autoantibodies against the nicotinic acetylcholine receptor (AChR) on the postsynaptic membrane at the neuromuscular junction. ${ }^{4,5}$ It was first described by Thomas Willis (1672), and more than two centuries later another patient with bulbar and limb muscle weakness who died ofrespiratory failure was reported. 6,7

There are various types of classification of Myasthenia gravis. It has been classified according to the age of onset, presence or absence of anti-AchR antibodies, severity, and the aetiology of the disease. Based on the age of onset age of onset Myasthenia gravis can be classed as transient neonatal or adult autoimmune. Transient neonatal myasthenia gravis is due to transfer of maternal anti-AchR antibodies through the placenta to the newborn reacting with the AChR of the neonate.

On the basis of presence or absence of anti-AChR antibodies Myasthenia gravis can be classed as seropositive or seronegative. Seropositive is the commonest type of acquired autoimmune myasthenia gravis accounting for up to $85 \%$ of patients with generalised myasthenia and $50 \%$ $60 \%$ with ocular myasthenia gravis test positive for antiAChR antibodies by radioimmunoassay. The rest 10\%-20\% of patients with acquired myasthenia gravis do not have anti-AChR antibodies detectable by radioimmunoassay. A subgroup of these patients have antibodies that bind to MuSK ${ }^{8}$ It has been proposed that the presence of antibodies against MuSK appears to define a subgroup of patients with seronegative myasthenia gravis who have predominantly localised, in many case bulbar, muscle weaknesses, reduced response to conventional immunosuppressive treatments, and muscle wasting. ${ }^{9}$ Essentially, seronegative myasthenia gravis is likely to be an autoimmune disorder involving antibodies against one or more components of the neuromuscular junction that are not detected by the current anti-AchR radioimmunoassay. Other distinct humoral factors are also being implicated are IgG antibodies that reversibly inhibit AChR function and a non-IgG (possibly IgM) factor that indirectly inhibits AChR function. ${ }^{10}$

Osserman's original classification divides adult myasthenia gravis into four groups based on the severity of the disease. $^{11}$

(1) Ocular myasthenia, where disease is confined to ocular muscles.

(2) Generalised myasthenia gravis of mild

(a) or moderate

(b) intensity.

(3) Severe generalised.

(4) Myasthenic crisis with respiratory failure.

But this classification has been modified by an ad hoc committee of the American myasthenia gravis foundation to standardise it for research purposes into following types: 12 
(I) Any ocular weakness; may have weakness of eye closure; strength of all other muscles being normal.

(II) Mild weakness other than ocular muscles, $+/ 2$ weakness of ocular muscles of any severity. IIa: predominant limb and/or axial involvement;

IIb: predominantly oropharyngeal and/or respiratory involvement.

(III) Moderate weakness affecting muscles other than ocular muscles, may have ocular weakness.

IIIa: predominant limb and/or axial involvement;

IIIb: predominantly oropharyngeal and/or respiratory involvement.

(IV) Severe weakness affecting muscles other than ocular muscles, may have ocular weakness.

IVa: predominant limb and/or axial involvement;

IVb: predominantly oropharyngeal and/or respiratory involvement.

(V) Defined by intubation with or without mechanical ventilation, except when employed during routine postoperative management. The use of feeding tube without intubation places the patient in class IVb

There are four classes based on the aetiology which are acquired autoimmune, transient neonatal (caused by the passive transfer of maternal anti-AChR antibodies), drug induced (D-penicillamine,curare, aminoglycosides, quinine, procainamide, and calcium channel blockers);

and congenital myasthenic syndromes (AChR deficiency, slow channel syndrome, and fast channel syndrome). ${ }^{13}$

Our patient was categorized to have II a. But our patient had no diurnal variation of symptoms. His lack of energy, weakness and difficulty in swallowing of food was actually the fatigue of MG. Being illiterate, the patient could not explain his symptoms properly and the physicians also did not evaluate those properly. It has to be remembered that it is not uncommon for a patient with MG to exhibit symptoms even of depression. ${ }^{14}$ The present case had atypical presentations initially although we found ptosis during examination. He had no diplopia which is a common complain in MG specially at evening. Such atypical cases can be confused with polymyositis, inclusion body myopathy, stroke, motor neuron disease. MG may be also confused with other conditions like neurasthenia, depression, hypothyroidism, myopathy, chronic motor polyneuropathy, Lambert-Eaton Myasthenic Syndrome (LEMS), chronic fatigue syndrome and so on. ${ }^{15}$ Thus, meticulous history taking, clinical examination and relevant laboratory investigations are required to rule out these possibilities.

Diagnosis of MG can be established by various tests which is guided by clinical consideration and country wise availability.

Among the serological tests employed for $\mathrm{MG}$, antiacetylcholine receptor $(\mathrm{AChR})$ antibody $(\mathrm{Ab})$ test is employed for diagnosing autoimmune myasthenia gravis (MG) with a specificity as high as $100 \%$, according to Padua et al). Results are positive in as many as $90 \%$ of patients who have generalized MG but in only $50-70 \%$ of those who have only ocular MG. But false negatives can occur in cases of purely ocular MG and false-positive anti-AChR Ab test results reported in cases of thymoma without MG,in patients with Lambert-Eaton myasthenic syndrome, small cell lung cancer, or rheumatoid arthritis treated with penicillamine, and in a small group of population older than 70 years. ${ }^{16}$ Antistriated muscle (anti-SM) Ab is present in about $84 \%$ of patients with thymoma in patients younger than 40 years, but less commonly in those without thymoma. So, a positive test result necessitates a search for thymoma in patients younger than 40 years. In individuals older than 40 years, thymoma can present without anti-SM Ab. Patients with negative results for anti-AChR Ab (seronegative $\mathrm{MG}$ ) can have positive test results for antibody to muscle-specific kinase (MuSK), a receptor tyrosine kinase.${ }^{16,17}$ Anti-MuSKpositive individuals tend to have more pronounced bulbar weakness and may have tongue and facial atrophy. They may have neck, shoulder and respiratory involvement without ocular weakness. They are also less likely to respond to acetylcholine esterase (AChE) inhibitors, and their symptoms may actually worsen with these medications. ${ }^{18 \text {, }}$ ${ }^{19}$ Unfortunately, we could not perform none of these tests due to lack of facilities.

Electrodiagnostic studies can demonstrate a defect of neuromuscular transmission and can aid in diagnosis of myasthenia. The following 2 studies are commonly performed:

- $\quad$ Repetitive stimulation of a muscle at 2-3 Hz, also known as repetitive nerve stimulation (RNS)

- Single-fiber electromyography (SFEMG), aimed at evaluating neuromuscular block, jitter, and fiber density

In patients with $\mathrm{MG}$, the number of AChRs is reduced, lowering the safety factor. During RNS, some EPSPs may not reach threshold, which means that no action potential is generated. This results in the decrement in the amplitude of the CMAP. In patients with myasthenia gravis, this decremental response usually has a maximum decrement at the fourth or fifth response, followed by a tendency toward 
repair. A stimulation rate of 1-5 per second should result in a $10 \%$ or more decrease in amplitude by the fourth or fifth action potential; any decrement over $10 \%$ is considered abnormal. Patients with MG rarely have a decremental response in a clinically normal muscle and so, testing a proximal weak muscle is a better yield than testing a unaffected distal muscle. Testing a facial muscle (eg, the orbicularis oculi) is useful because most patients suffer from eyelid weakness or ptosis. RNS results are less likely to be positive in patients with ocular MG. Repetitive Nerve Stimulation test was also done in our patient and it was consistent with Myasthenia Gravis (9.2 to $20.9 \%$ decremental responses). 16

Another option is Single-fiber electromyography. The singlefiber needle used in SFEMG, which has a small recording surface, allows recording from individual muscle fibers. The advantage of SFEMG is that treatment with AChR inhibitors does not normalize SFEMG results. Where RNS findings are abnormal in only 44-65\% of patients, SFEMG findings are abnormal in almost $100 \%$. SFEMG can be a good substitute for RNS in patients with ocular MG as shown by Padua et al (almost 100\% sensitivity). SFEMG has some disadvantages as is technically demanding, highly operator-dependent and, has a lower specificity, and it can give positive results in other neuromuscular disorders. 16

Another option is the Anticholinesterase Test is. It uses the principle that Edrophonium, a short-acting AChE inhibitor that improves muscle weakness in patients with MG. Evaluate weakness (eg, ptosis, partial or complete ophthalmoplegia, and forced hand grip) in an involved group of muscles before and after intravenous (IV) administration of edrophonium. Blinding of both the examiner and the patient increases the validity of the test. Sinus bradycardia due to excessive cholinergic stimulation of the heart is a serious complication; consequently, an ampule of atropine should be available at the bedside or in the clinic room while the test is performed.

This test has some fallacies and can give both false-negative results and false-positive results. It has a low sensitivity in ocular MG; $50 \%$ of patients presenting with eye symptoms will be missed. On the other hand, diseases other than MG, such as amyotrophic lateral sclerosis (ALS) and cavernous sinus lesions can score positive on the test. This test has been combined with electromyography (EMG) and ocular tonography to increase its sensitivity in ocular MG; however, it still produces false-negative and false-positive results. ${ }^{20}$

The ice pack test (ie, placing ice over the lid) has gained interest among ophthalmologists for assessing improvement in ptosis and diplopia in ocular MG. The rationale behind this test is that cooling might improve neuromuscular transmission. The validity of such a test has been questiontioned by various experts demonstrating that patients with ocular MG actually improve on the ice, heat, and modified sleep tests. Hence, rest might be the cause of the improvement in ocular signs. Both the ice test and the rest test are sensitive and specific in ocular MG. ${ }^{21,22}$

Some other tests are recommended. Testing for rheumatoid factor and antinuclear antibodies (ANAs) is indicated to rule out systemic lupus erythematosus (SLE) and rheumatoid arthritis (RA), thyroid function tests to rule out associated Graves disease or hyperthyroidism. This is essential, especially in patients with ocular MG where the concomitant hyperthyroidism is most frequent.

Chest $\mathrm{X}$ ray, $\mathrm{A} / \mathrm{P}$ and lateral views may identify a thymoma as an anterior mediastinal mass. A negative chest radiograph does not rule out a smaller thymoma, in which case a chest computed tomography (CT) scan is required. Chest CT scan is mandatory to identify or rule out thymoma or thymic enlargement in all cases of MG. This is especially true in older individuals.

In our patient, though chest $\mathrm{x}$ ray did non initially give clinical impression of thymoma but ultimately histopathological tests ruled in favour of a thymoma.

Thymoma is a rare tumour which may be related to MG in about 10 to $15 \%$ of cases. Thymoma is common in middle age and elderly male (50-60yrs). ${ }^{2-3}$ As our patient was middle aged male we suspected to be a case of thymoma, even though imaging reports were normal. Ultimately biopsy report confirmed the presence of thymoma. A thymoma, which is an epithelial tumor of the thymus gland that is usually benign, occurs in about 10 to $15 \%$ of adult patients with MG. $30 \%$ patients with thymoma are associated with MG. ${ }^{3}$ Thymoma is a rare disease with incidence rate of 32/1.000.000/year. ${ }^{4,5}$ Although thymectomy may improve the myasthenic symptoms, MG can develop from months to years after the removal of a thymoma in previously nonmyasthenic patients. . The histological classiûcation of thymoma has remained a subject of controversy for many years. In 1999, the World Health Organization (WHO) Consensus Committee published a histological typing system for tumors of the thymus. ${ }^{5-9}$

The major Histological Classifications of Thymoma by WHO is-

A - Medullary

AB - Mixed type

B1 - Predominant cortical

B2 - Cortical

B3 - Well differentiated thymic carcinoma

C - Undifferentiated carcinoma. 
The cardinal symptom of MG is abnormal fatigue of the muscles. Movement although initially strong rapidly weakens. Intensification of symptoms occurs towards the end of the day or following exercise. Ocular motor disturbance (50-66\%) are the first symptoms with ptosis or diplopia but ultimately it is present in $>90 \%$ cases 10 . Bulbar presentation occurs in $5-10 \%$ cases but ultimately it is present in $80 \%$ of cases. Initial presentation with limb weakness is uncommon $(10 \%)$. In the limbs, most commonly shoulder girdle is affected with difficulty with over-head tasks. Sometimes pelvic girdle (difficulty with getting out of chairs) may be affected. Respiratory muscle may be involved with respiratory paralysis and death. A patient requiring mechanical ventilation due to severe respiratory weakness is said to be in crisis. ${ }^{30}$

Ptosis may be unilateral or bilateral. Patients with mild diplopia may initially seek the help of an ophthalmologist. Myasthenic weakness may mimic third, fourth, and sixth cranial nerve palsies as well as an internuclear ophthalmoplegia. MG never affects pupillary function. Difficulty in chewing, speaking, or swallowing may also be the cause for initial presentation, but the occurrence of these symptoms is less frequent than the aforementioned ocular symptoms. ${ }^{12}$ These patients usually present to ENT specialist. The symptoms of MG are worsened at the end of the day or after repetitive activities of involved muscles. Examination of a patient with $\mathrm{MG}$ therefore is directed at muscle strength and demonstrating pathologic fatigability. A few maneuvers that may be used are having the patient look up for several minutes (examining for ptosis or extraocular weakness), counting aloud to 100 (listening for nasal or slurred speech), or by repetitively testing the proximal muscles. ${ }^{3,14}$ The results for the remainder of the neurologic examination are usually normal. A key point to remember is that if a patient has generalized limb weakness without ocular involvement, the diagnosis of MG should be questioned. ${ }^{14}$

Clinical data suggest that patients with thymoma associated MG have high-grade symptoms with low rate of remission even after therapy. ${ }^{30}$ But our patient had mild symptoms despite having thymoma and responded well after taking low dose of pyridostigmine. Although the improvement after thymectoy is usually delayed, our patient had clinical improvement within weeks after thymectomy.

\section{Conclusion:}

It is not difficult to diagnose a case of MG with typical symptoms. But it may be very difficult when symptoms are bizarre and vague. Patient may be undiagnosed despite consulting with physicians repeatedly. High degree of clinical suspicion is therefore necessary to diagnose these cases.
Although patients with thymoma associated MG have highgrade symptoms with low rate of remission after therapy; patient may have milder symptoms with rapid improvement after therapy.

\section{Conflict of Interest : None}

\section{References:}

1. Blossom GB, Ernstoff RM, Howells GA, Bendick PJ, Glover JL. Thymectomy for myasthenia gravis. Arch Surg 1993; 128: 855-62.

2. Evoli A, Batocchi AP, Zelano G, et al. Familial autoimmune gravis: report of four families. JNNP 1995; 58:729-31.

3. Keesey JC. Clinical evaluation and management of myasthenia gravis. Muscle Nerve 2004; 29:484-505

4. Drachman D. Myasthenia gravis. In: Rose N, Mackay I, eds. The autoimmune diseases. 3rd Ed. San Diego: Academic Press, 1998:637-62.

5. Tzartos SJ, Barkas T, Cung MT, et al. Anatomy of the antigenic structure of a large membrane autoantigen, the muscle-type nicotinic acetylcholine receptor. Immunol Rev 1998;163:89-120.

6. Willis T. De anima brutorum. Oxford, UK: Theatro Sheldoniano, 1672:404-6.

7. Wilks S. On cerebritis, hysteria and bulbar paralysis, as illustrative of arrest of function of the cerebrospinal centres. Guys Hosp Rep 1877;22:7-55.

8. Hoch W, McConville J, Helms S, et al. Auto-antibodies to the receptor tyrosine kinase MuSK in patients with myasthenia gravis without acetylcholine receptor antibodies. Nature Med 2001;7:365-8.

9. Vincent A, Bowen J, Newsom-Davis J, et al. Seronegative generalized myasthenia gravis: clinical features, antibodies, and their targets. Lancet Neurology 2003;2:99-106.

10. Update on myasthenia gravis .B R Thanvi, T C N Lo. Postgrad Med J 2004;80:690-700

11. Osserman KE. Myasthenia gravis. New York, NY: Grune and Stratton, 1958

12. Jablecki A 3rd, Barohn RJ, Ernstoff RM, et al. Myasthenia gravis: recommendations for clinical research standards. Task Force of the Medical Scientific Advisory Board of the Myasthenia Gravis Foundation of America. Neurology 2000;55:16-23

13. Penn AS, Low BW, Jaffe JL, et al. Drug-induced autoimmune myasthenia gravis. Ann N Y Acad Sci 1998; 841:433-49.

14. Massey JM. Acquired myasthenia gravis [review]. Neurol Clin.1997; 15:577-595.

15. Adams and Victor's Principles of Neurology, $9^{\text {th }}$ edition: page 1412 . 
16. Padua L, Stalberg E, LoMonaco M, Evoli A, Batocchi A, Tonali P. SFEMG in ocular myasthenia gravis diagnosis. Clin Neurophysiol. Jul 2000;111(7):1203-7.

17. Tindall RS. Humoral immunity in myasthenia gravis: biochemical characterization of acquired antireceptor antibodies and clinical correlations. Ann Neurol. Nov 1981;10(5):437-47.

18. Pasnoor M, Wolfe GI, Nations S, Trivedi J, Barohn RJ, Herbelin L, et al. Clinical findings in MuSK-antibody positive myasthenia gravis: a U.S. experience. Muscle Nerve. Mar 2010;41(3):370-4

19. Sanders DB, El-Salem K, Massey JM, et al. Clinical aspects of MuSK antibody positive seronegative MG. Neurology. Jun 24 2003;60(12):1978-80

20. Phillips LH 2nd, Melnick PA. Diagnosis of myasthenia gravis in the 1990s. Semin Neurol. Mar 1990;10(1):62-9

21. Movaghar M, Slavin ML. Effect of local heat versus ice on blepharoptosis resulting from ocular myasthenia. Ophthalmology. Dec 2000;107(12):2209-14

22. Benatar M. A systematic review of diagnostic studies in myasthenia gravis. Neuromuscul Disord. Jul 2006;16(7):45967
24. de Jong et al. Eur. J. Cancer, 441, 123,2008.

25. Rosai J. Histological Typing of Tumors of the Thymus. 2nd ed.Berlin, Germany: Springer-Verlag; 1999. World Health Organization International Histological Classification of Tumors.

26. Rowland LP, Aranow Jr H, Hoefer PFA. Myasthenia gravis appearing after the removal of thymoma. Neurology 1957;7:584-588.

27. Namba T, Brunner NG, Grob D. Myasthenia gravis in patients with thymoma, with particular reference to onset after thymectomy. Medicine (Baltimore) 1978; 57:411-433.

28. Hassel B, Gilhus NE, Aarli JA, Skogen OR. Fulminant myasthenia gravis and polymyositis after thymectomy for thymoma. Acta Neurol Scand 1992; 85:63-65.

29. Mineo TC, Biancari F, D'Andrea V. Late onset of myasthenia gravis after total resection of thymoma: report of two cases. J Cardiovasc Surg (Torino) 1996; 37:531-533

30. Grob D, Arsura EL, Brunner NG, Namba T. The course of myasthenia gravis and therapies affecting outcome.Ann $\mathrm{N}$ Y Acad Sci 1987; 505: 472-99 